\title{
Fallopian Tube Gancer: A Rare Entity
}

Neema Sharma', Urvashi Prasad Jha', Ramandeep', Abha Sabhikhi', Ritambhara' ${ }^{1}$, Priyata'

Department of Minimal and Natural Access Gynae \& Gynae Cancer Surgery' and Department of Histopathology ${ }^{2}$, Fortis Flt. Lt. Rajan Dhall Hospital, Vasant Kuni, New Delhi, India.

Abstract:

Primary fallopian tube cancer is a rare entity in patients who present with bilateral hydrosalpinx. We present a case of bilateral hydrosalpinx with pain. We did laparoscopic salpingectomy with frozen section which revealed malignancy. This was followed by staging laparotomy. Strong index of suspicion is required in patients with hydrosalpinx for the efficient management of the patient.

Key words: Fallopian Tube Neoplasms, Salpingectomy, Salpingitis, Laparatomy, Pain.

\section{Introduction}

Primary fallopian tube carcinoma (PFTC) is an uncommon tumor accounting for approximately $0.14 \%-1.8 \%$ of female genital malignancies. Preoperative diagnosis is rare in the range of $0 \%-10 \%$ [1]. We present a rare case of primary fallopian tube cancer.

\section{Case Report}

PIL1, 48 years old perimenopausal woman presented with severe pain lower abdomen for one day with a 3 month history of dull aching pain. The patient gave a history of longstanding pelvic inflammatory disease. On examination, a $10 \times 10$ $\mathrm{cm}$ mass was felt in the right fornix. Ultrasound and CT scan revealed a $13 \times 5 \mathrm{~cm}$ elongated tubular structure in the right adnexa and $6 \times 1.8 \mathrm{~cm}$ tubular structure in the left fornix ?hydrosalpinx with multiple calcified periportal and portacaval lymph nodes and no free fluid. Tumor markers were normal. The patient was counseled for laparoscopic salpingo-ophorectomy with frozen section and a view to proceed with staging if positive for malignancy. Laparoscopic findings revealed tense elongated $10 \times 5 \mathrm{~cm}$ right adnexal mass with right ovary not seen separately [Fig.1], left hydrosalpinx $4 \times 4 \mathrm{~cm}$ and enlarged left ovary $4 \times 4 \mathrm{~cm}$ with cystic changes [Fig.2]. On mobilizing the mass, pus was seen [Fig.3]. Laparoscopic bilateral salpingo-ophorectomy was performed and sent for frozen section which revealed malignancy. Total abdominal hysterectomy with pelvic lymphadenectomy with omentectomy and appendectomy was performed.

\section{Corresponding Author: Dr. Urvashi Prasad Jha}

Email: urvashipjhaclinic@gmail.com

Received: March 3, 2015 | Accepted: April 21, 2015 | Published Online: May 15, 2015

This is an Open Access article distributed under the terms of the Creative Commons Attribution License (creativecommons.org/licenses/by/3.0)

Conflict of interest: None declared | Source of funding: Nil | DOl: http://dx.doi.org/10.17659/01.2015.0053 
Gross description of the right adnexa showed a $13 \times 4 \times 3 \mathrm{~cm}$ mass comprising of fallopian tube with an adherent ovary. Inner surface showed firm to hard, grey areas with papillary excrescences. Fimbrial end of the tube was closed. Cut sections of the ovary revealed irregular grey, white area. The left fallopian tube was dilated and $7 \mathrm{~cm}$ in length. Cut section revealed small papillary excrescences. Fimbrial end was closed and left ovary showed hemorrhagic areas.

Histopathology revealed bilateral papillary serous adenocarcinoma of fallopian tubes with high grade, tumor arising from the lining, extending into the wall of the fallopian tubes. Serosal extension was not seen, the lymph vascular invasion was not present [Fig.4,5]. Uterus, bilateral ovaries, appendix, omentum and lymph nodes were not involved by tumor. Immunohistochemistry revealed WT 1 positive in tumor cell and CK 7 positive in tumor cells. Peritoneal fluid for cytology was negative. It was FIGO-1c, TNM (TlcNOMO). IV taxane/ carboplatin was recommended for 3-6 cycles in the tumor board meeting.

\section{Discussion}

The aetiology of PFTC is unknown, but nulliparous women, women with germ-line BRCA mutation and women in certain occupations such as smelting workers, artistic workers, hairdressers, packers, nurses, shop workers, and clerical workers appear to be at increased risk of PFTC [2]. The median age of occurrence of 55 years [3]. There are no specific symptoms. Latzko's triad of symptoms has been reported in $15 \%$ of cases to diagnose PFTC. It consists of intermittent profuse serosanguinous vaginal discharge, colicky pain relieved by discharge, and the presence of an abdominal or pelvic mass [4]. It is often diagnosed early because of abdominal pain secondary to tubal distention.

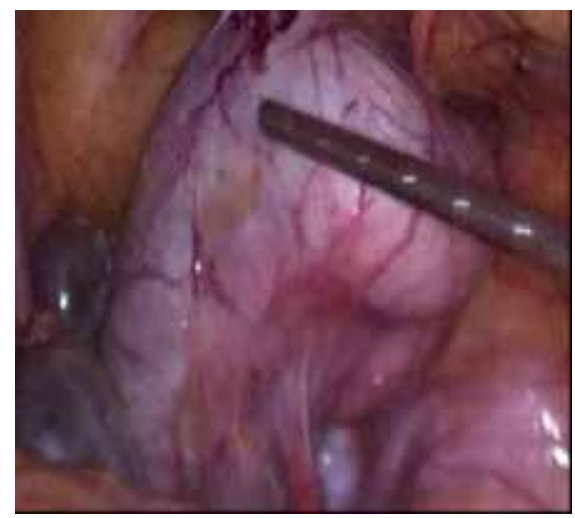

Fig.1: Tense elongated $10 \times 5 \mathrm{~cm}$ right adnexal mass, right ovary not seen separately.

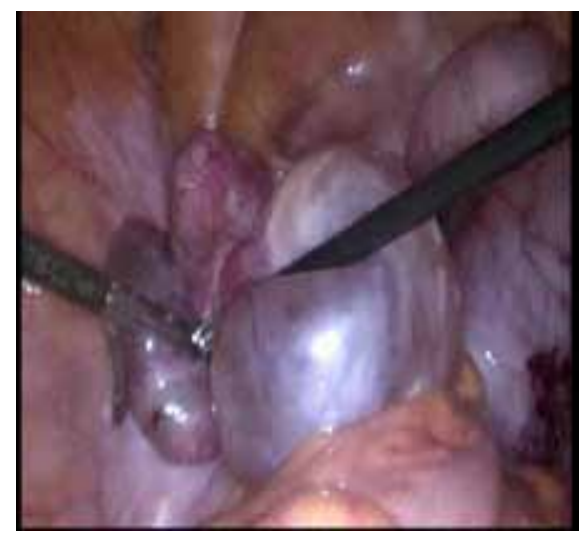

Fig.2: Left hydrosalpinx $4 \times 4 \mathrm{~cm}$ and $4 \times 4 \mathrm{~cm}$ cystic, enlarged left ovary.

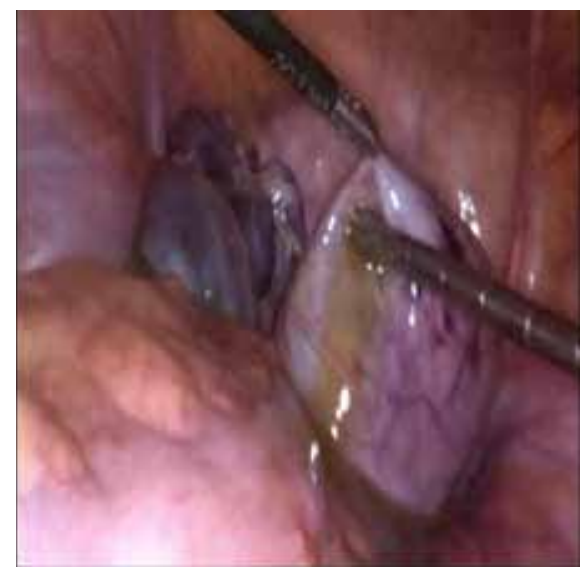

Fig.3: Pus aspirated from the right tubal mass. 

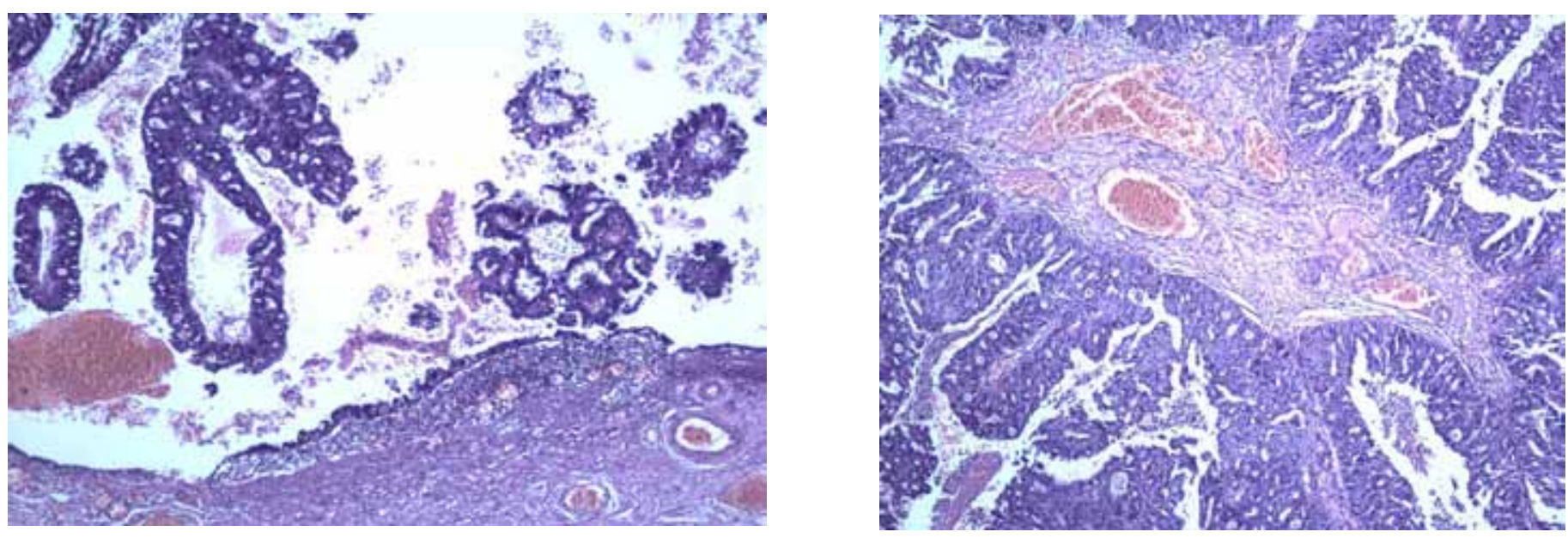

Fig.4,5: Tumor arising from the lining epithelium, complex arborizing papillae and prominent nucleoli.

Ultrasonographer should have a high level of suspicion for the PFTC in older women with hydrosalpinx. Transvaginal ultrasound examination with color Doppler can detect areas of neovascularization within the Fallopian tube. Although CA-1 25 per se is not diagnostic for PFTC, $>80 \%$ of patients have elevated pre-treatment serum CA-125 levels [5].

It is difficult to differentiate PFTC from epithelial ovarian cancer. Histopathologist have strict criteria to diagnose PFTC [6]: (a) main tumor is in the tube and arises from the endosalpinx; (b) histologically, the pattern reproduces the epithelium of the mucosa and often shows a papillary pattern; (c) if the wall is involved, the transition between benign and malignant epithelium should be demonstrable; and (d) ovaries and endometrium are either normal or contain less tumor than the tube.

Tubal carcinoma spreads in much the same manner as epithelial ovarian cancer, principally by the transcelomic exfoliation of cells, can also occur by means of contiguous invasion and hematogenous dissemination [7]. Bilateral tubal involvement has been reported in 10\%-27\% of cases [8]. The staging of PFTC is based on the surgical findings at laparotomy. The International Federation of Gynecology and Obstetrics (FIGO) classification of epithelial ovarian cancer is used for PFTC.

Surgical principles are the same as those used for ovarian cancer while platinum-based combination chemotherapy is the most commonly used adjuvant therapy for these patients, identical to epithelial ovarian cancer patients.

\section{Conclusion}

PFTC is a rare tumor accounting for $<1 \%$ of all female genital tract cancers and should be kept in mind when older women present with hydrosalpinx.

\section{References}

1. Riska A, Leminen A, Pukkala E. Sociodemographic determinants of incidence of primary fallopian tube carcinoma, Finland 1953-97. Int J Cancer. 2003; 104:643-645.

2. Vicus D, Finch A, Rosen B, et al. Risk factors for carcinoma of the fallopian tube in women with and without a germline BRCA mutation. Gynecologic Oncology. 2010;1 18(2):155-159. 
3. Sedlis A. Carcinoma of the fallopian tube. Surg Clin North Am. 1978;58:121-129.

4. Ajithkumar TV, Minimole $\mathrm{AL}$, John $M M$, Ashokkumar OS. Primary fallopian tube carcinoma. Obstet Gynecol Surv. 2005;60:247252.

5. Katke RD, Gadekar S, Pagare P. A Rare Case of Carcinoma of Ovary with Carcinoma of Cervix. Journal of Case Reports. 2014;4(1):217-220.
6. Hu CY, Taymor ML, Hertig AT. Primary carcinoma of the fallopian tube. Am J Obstet Gynecol. 1950;59:58-67.

7. Yoonessi M. Carcinoma of the fallopian tube. Obstet Gynecol Surv. 1979;34:257-270.

8. Schiller HM, Silverberg SG. Staging and prognosis in primary carcinoma of the fallopian tube. Cancer. $1971 ; 28: 389-395$. 